\title{
Ultra-sonografia intra-operatória (USIO)
}

\author{
Márcio Martins Machado \\ Médico Radiologista, Pós-graduando do \\ Departamento de Radiologia da FMUSP
}

Desde os primeiros estudos sobre a USIO, realizados no início da década de 60 por Schlegel et al. (1961) e por Knight e Newell (1963), esta modalidade de diagnóstico por imagem vem passando por aprimoramento constante e ganhando espaço entre os métodos de imagem. Esses primeiros estudos visavam identificar cálculos renais pela ultra-sonografia, durante cirurgias abdominais para sua retirada ${ }^{(\mathbf{1})}$, ou identificar coledocolitíases durante colecistectomias ${ }^{(2)}$. Essas experiências iniciais utilizavam a ultra-sonografia em modo-A, não obtendo ampla aceitação no ambiente cirúrgico.

Com o surgimento da ultra-sonografia de melhor qualidade em modo-B, no final da década de 70 , houve um ressurgimento do interesse para essa modalidade (a USIO) de exame. Dessa forma, eram utilizados transdutores de 5 e 7,5 $\mathrm{MHz}$ para o exame intra-operatório, e a representatividade das imagens passou a ter grande aceitação pelos cirurgiões. Nesse sentido, o método foi ganhando ampla aceitação, inicialmente no Japão e na Europa, especialmente da metade para o final da década de 70 , sendo que, posteriormente, a partir de meados da década de 80 , ampliou-se sobremaneira sua utilização nos $\operatorname{EUA}^{(3-5)}$.

A evolução continuou na década de 90 , com a ampliação da utilização da USIO, agora com novas aquisições, como a utilização do Doppler, o que possibilitou aumentarem-se as possibilidades de estudo das patologias abdominais, durante o exame intra-operatório. Nessa mesma década, com a expansão da utilização da vídeo-laparoscopia, a USIO acompanhou esse desenvolvimento mediante o aprimoramento da ultra-sonografia intra-operatória por vídeo-laparoscopia (USIO-LAPA). Em nosso meio, foram publicados trabalhos orientando sobre o desenvolvimento técnico da USIO-LAPA ${ }^{\left({ }^{()}\right.}$e sobre alguns aspectos metodológicos que poderiam ser usados para melhorar a capacidade de estadiamento do fígado, durante vídeo-laparoscopias para manuseio de neoplasias abdominais ${ }^{(7)}$.

Nos anos 90 vemos uma expansão exuberante das aplicações da USIO durante as cirurgias abdominais, em especial no estudo do fígado, para a identificação de nódulos, e do pâncreas, para a identificação de tumores neuroendócrinos ou outros tumores de baixa agressividade (como tumores císticos serosos-microcísti$\cos )^{(5-15)}$. Nesse sentido, os médicos imagenologistas devem estar atentos aos aspectos concretos e coerentes do método (da USIO) nos dias atuais, pois os especialistas multidisciplinares que acompanham esses pacientes estão cada vez mais requisitando os exames de USIO e esperando deles os mesmos resultados que vêm sendo publicados e apresentados em congressos no Japão, Europa e América do Norte.

Primeiro, a grande maioria dos estudos publicados mostra de forma bastante clara que a USIO apresenta maior sensibilidade e especificidade (e acurácia) na identificação de nódulos hepáticos, quando comparada com os exames pré-operatórios, assim como demonstra de forma mais dinâmica, no próprio ambiente cirúrgico, as relações vasculares dos tumores hepáticos ${ }^{(\mathbf{5})}$. Esses dois aspectos têm contribuído para a mudança de conduta no intra-operatório em cerca de $31 \%$ a $51 \%$ dos casos de pacientes com nódulos hepáticos que serão submetidos a laparotomia para ressecções do fígado, conforme demonstrado por grandes séries publicadas na literatura ${ }^{(5)}$. Outro aspecto que deve ser reconhecido é que a USIO é utilizada como "padrão ouro" nos estudos que buscam avaliar a capacidade diagnóstica de nódulos hepáticos, quer pela ultra-sonografia, tomografia computadorizada (convencional ou helicoidal) ou ressonância magnética, como demonstrado em estudos no exterior ou em nosso meio ${ }^{(8,9,16,17)}$.

Segundo, no caso dos tumores pancreáticos (especialmente dos insulinomas e outros tumores de baixa agressividade), também a USIO vem apresentando capacidade diagnóstica superior àquela dos métodos pré-operatórios, inclusive quando comparada com a ultra-sonografia endoscópica (US-endoscópica) ${ }^{(5,18,19)}$. Esses casos são especialmente evidentes para lesões menores que $0,8 \mathrm{~cm}$ e localizadas no corpo pancreático distal e cauda pancreática. Outro aspecto de interesse é que a USIO permite que se identifique, no campo operatório, a posição do ducto de Wirsung e se estabeleça a sua relação com o tumor pancreático. Dessa forma, tumores pancreáticos menos agressivos (como os insulinomas) podem ser ressecados sem comprometer o ducto de Wirsung, permitindo a preservação da máxima quantidade de parênquima pancreático normal possível, o que diminui a morbidade e a mortalidade operatória desses procedimentos ${ }^{(10,18,19)}$.

Evidentemente, a USIO não é um exame pré-operatório, apresentando, por isso, limitação no seu uso e, dessa forma, também não concorrendo com os outros exames no algoritmo de investigação das afecções abdominais. A USIO se beneficia sobremaneira das informações fornecidas pelos exames de ultra-sonografia abdominal, tomografia computadorizada convencional ou 
helicoidal e de ressonância magnética, atuando como complementar destes, naquele pequeno grupo de pacientes que submeter-se-ão a laparotomias para o tratamento de alterações orgânicas específicas, como já referido.

Entende-se, portanto, o máximo de relacionamento que deve existir entre as informações fornecidas pelos exames pré-operatórios e que serão utilizadas pela USIO durante o exame intraoperatório, para que se maximize os benefícios dos métodos de imagem como um todo, buscando o maior beneficiário, que é o paciente que será operado. Ademais, a USIO representa a interface mais dinâmica entre imagenologistas e cirurgiões (e clíni$\cos$ ), dependendo, para a qualidade dessa inter-relação, do conhecimento amplo dessa emergente modalidade de exame de imagem pelos radiologistas.

\section{REFERÊNCIAS}

1. Schlegel JU, Diggdon P, Cueltar J. The use of ultrasound for localizing renal calculi. J Urol1961;86:367-9.

2. Knight PR, Newell JA. Operative use of ultrasonics in cholelithiasis. Lancet 1963;i:1023-5.

3. Cerri LMO, Cerri GG. Intraoperative ultrasonography of liver, bile ducts and pancreas. Rev Paul Med 1996;114:1196-207.

4. Cerri LMO. Contribuição da ultra-sonografia intra-operatória em fígado, vias biliares e pâncreas. [Tese de doutorado]. São Paulo: Universidade de São Paulo, 1995.

5. Luck AJ, Maddern GJ. Intraoperative abdominal ultrasonography. Br J Surg 1999;86:5-16.

6. Machado MM, Oliveira IRS, Cerri GG. Considerações sobre a evolução técnica dos transdutores na ultra-sonografia intra-operatória vídeo-laparoscópica (USIO-LAPA). Radiol Bras 1999;32:85-7.

7. Machado MM, Cerri GG. Proposta de metodização da ultra-sonografia intra-operatória vídeo-laparoscópica (USIO-LAPA) no estadiamento do fígado em pacientes portadores de neoplasia gastrointestinal. Radiol Bras 1998;31:375-7.

8. Machado MM, Cerri GG, Oliveira IRS, et al. Contribuição da ultra-sonografia intra-operatória (USIO) no estudo de pequenas imagens nodulares hipo- atenuantes identificadas à tomografia computadorizada (TC) no exame préoperatório de pacientes com adenocarcinoma colorretal e de pâncreas. Comunicação original. Radiol Bras 1999;32:255-8.

9. Machado MM, Cerri GG, Oliveira IRS, et al. Contribuição da ultra-sonografia intra-operatória (USIO) no estudo de pequenas imagens nodulares hipoatenuantes identificadas à tomografia computadorizada (TC) no exame préoperatório de pacientes com adenocarcinoma colorretal e de pâncreas. Radiol Bras 2000;33(supl. $\mathrm{n}^{\circ}$ 1):3.

10. Machado MM, Saito OC, Oliveira IRS, Penteado S, Machado MCC, Cerri GG. Tumor de Frantz: características anátomo-sonográficas à ultra-sonografia intra-operatória (USIO) e implicações no manuseio cirúrgico. Nota prévia. Radiol Bras 1999;32:89-92.

11. Machado MM, Cerri GG. Ultra-sonografia intra-operatória (USIO) do fígado. In: Saad WA, D'Albuquerque LAC, Chaib E, eds. Atlas de cirurgia hepática. São Paulo: Ateneu, 1999:191-8.

12. Machado MM, Oliveira IRS, Saito OC, Cerri GG. Ultra-sonografia intraoperatória (USIO) do fígado. Radiol Bras 2000;33:15-8.

13. Machado MM, Rosa ACF, Cerri GG. Ultra-sonografia intra-operatória (USIO). In: Habr-Gama A, Rodrigues JG, Cecconello I, et al., eds. Atualização em cirurgia do aparelho digestivo e em coloproctologia. São Paulo: Frontis Editorial, 2000:179-94.

14. Machado MM, Rosa ACF, Machado MCC, Penteado S, Cerri GG. Contribuição da ultra-sonografia intra-operatória (USIO) no estudo das características anátomo-sonográficas do leiomiossarcoma primário de pâncreas. Nota prévia. Radiol Bras 2001;34:295-7.

15. Machado MM, Rosa ACF, Herman P, Saad WA, Cerri GG. Adenomatose hepática múltipla. Diagnóstico pela ultra-sonografia intra-operatória (USIO). Nota prévia. Radiol Bras 2001;34:369-72.

16. Seneterre E, Taourel P, Bouvier Y, et al. Detection of hepatic metastases: ferumoxides-enhanced MR imaging versus unenhanced MR imaging and CT during arterial portography. Radiology 1996;200:785-92.

17. Faria SC. Estudo comparativo da eficácia da tomografia computadorizada com contraste endovenoso com a ressonância magnética sem contraste na detecção de lesões hepáticas focais em pacientes com neoplasia colorretal. [Tese de mestrado]. São Paulo: Universidade Federal de São Paulo-Escola Paulista de Medicina, 2000.

18. Huai JC, Zhang W, Niu HO, Su ZX, McNamara JJ, Machi J. Localization and surgical treatment of pancreatic insulinomas guided by intraoperative ultrasound. Am J Surg 1998;175:18-21.

19. Machado MCC, Jukemura J, Penteado S, et al. Tratamento cirúrgico dos insulinomas - estudo de 59 casos. Rev Ass Med Bras 1998;44:159-66. 